Research Article

\title{
Contamination and Antimicrobial Susceptibility Testing of Staphylococcus aureus Isolated from Pork in Fresh Markets, Nongchok District, Thailand
}

\author{
Doungjit Kanungpean ${ }^{(D)},{ }^{1}$ Shinji Takai, ${ }^{2}$ and Tsutomu Kakuda ${ }^{2}$ \\ ${ }^{1}$ Faculty of Veterinary Medicine, Mahanakorn University of Technology, 140 Cheum-Sampan Rd., Nongchok, \\ Bangkok 10530, Thailand \\ ${ }^{2}$ Laboratories of Animal Hygiene, School of Veterinary Medicine, Kitasato University, Higashi 23-351, Towada, \\ Aomori 034-8628, Japan
}

Correspondence should be addressed to Doungjit Kanungpean; doungjit@mut.ac.th

Received 16 November 2020; Revised 14 February 2021; Accepted 25 February 2021; Published 8 March 2021

Academic Editor: Carlos Gonz lez Rey

Copyright (c) 2021 Doungit Kanungpean et al. This is an open access article distributed under the Creative Commons Attribution License, which permits unrestricted use, distribution, and reproduction in any medium, provided the original work is properly cited.

We surveyed Staphylococcus aureus contamination in 110 pork samples from 12 fresh meat markets in Nongchok district, Bangkok, Thailand, and performed antimicrobial susceptibility testing with the disk diffusion method. The prevalence of $S$. aureus was $28.18 \%$, and 52 strains were isolated. Antimicrobial susceptibility testing using the disk diffusion method revealed that $80.77 \%$ of the isolates were resistant to tetracycline and $76.92 \%$ to ampicillin. All strains were $100 \%$ susceptible to cloxacillin, cefoxitin, gentamicin, and cefazolin. The high percentage of antibiotic resistance to tetracycline and ampicillin was attributed to their use in treating infections in farmed animals and their addition to animal food for disease prevention. Interestingly, the present study revealed the intermediate resistance of $S$. aureus (13.46\% of $S$. aureus-positive pork samples) to vancomycin which is a common medicine for treating severe infection in humans, suggesting that the trend of resistance might increase and becoming a serious problem of public health for both humans and animals.

\section{Introduction}

Staphylococcus aureus is a Gram-positive coccus bacterium that tends to be arranged in grape-like clusters. This bacterium can grow in a medium containing up to $10 \%$ salt and is coagulasepositive [1]. S. aureus is found on the skin and mucous membranes of animals and humans and is also an environmental contaminant [2]. However, in developing countries such as Thailand, where meat is sold at fresh meat markets, the meat may be contaminated by organisms such as S. aureus, Escherichia coli, and Campylobacter jejuni [2-4]. S. aureus is the most common bacterial infection in humans and animals [1]. It can colonize humans and is the leading cause of bacteremia, infective endocarditis, and skin and soft tissue disease (e.g., impetigo and folliculitis); $S$. aureus also causes invasive infections and toxin-mediated diseases $[1,5]$. Multiple antimicrobial-resistant pathogenic bacteria have been recognized by the World Organization for Animal Health, the Food and Agriculture
Organization, and the World Health Organization as an important public health issue $[6,7]$. Furthermore, S. aureus has shown antimicrobial resistance in human and animal therapy [3], and strains of methicillin-resistant S. aureus (MRSA) are important human pathogens. The spread of MRSA has largely focused on pigs because of widespread worldwide reports of MRSA colonization and antimicrobial resistance in pigs [4]. Our study aimed to determine the extent of $S$. aureus contamination and the antimicrobial susceptibility of subsequent isolates in pork sold in fresh meat markets in Thailand.

\section{Materials and Methods}

2.1. Sample Collection and S. aureus Identification. Hundred and ten pork samples were collected randomly for analyzing from 12 markets in Nongchok district, Bangkok, Thailand. Sample amounts of the market no. 1 to no. 12 were 5 , $20,20,10,5,5,5,5,5,10,5$, and 15 , respectively. The samples 
were kept at $4^{\circ} \mathrm{C}$ before processing for culture. Each sample was cut into small pieces, placed in approximately $90 \mathrm{ml}$ of buffered peptone water (Himedia Laboratories, Mumbai, India) and mixed for $3 \mathrm{~min}$. Then, $10 \mu \mathrm{l}$ of the suspension was plated onto Baird-Parker agar (Himedia Laboratories, Mumbai, India) and incubated at $37^{\circ} \mathrm{C}$ for $18-24 \mathrm{hr}$. S. aureus grows as a black and shiny colony, with a fine white rim surrounded by a clear zone on Baird-Parker agar. All colonies identified as $S$. aureus were confirmed by a positive result for coagulase $[2,8]$.

2.2. Antimicrobial Susceptibility Testing. Disk diffusion antimicrobial susceptibility tests were performed on the confirmed S. aureus isolates. The common used antibiotics for treating respiratory disease in farm animals were chosen for the antimicrobial susceptibility tests, including ampicillin $(10 \mu \mathrm{g})$, cloxacillin $(5 \mu \mathrm{g})$, cefoxitin $(30 \mu \mathrm{g})$, cefazolin $(30 \mu \mathrm{g})$, gentamicin $(10 \mu \mathrm{g})$, vancomycin $(30 \mu \mathrm{g})$, and tetracycline $(30 \mu \mathrm{g})$ (Oxoid Ltd., Hampshire, UK). Briefly, S. aureus colonies were picked and subcultured by streaking on nutrient agar (Himedia Laboratories, Mumbai, India) and incubating the plate at $37^{\circ} \mathrm{C}$ for 18-24 hr. Then, a sterilized loop was used to pick four or five colonies from the nutrient agar and they were suspended in $2 \mathrm{ml}$ of Mueller-Hinton broth (Difco Laboratories, Inc., NJ, USA). After adjusting to a turbidity of $0.5 \mathrm{McF}$ arland standards, a sterile swab was dipped into the tube and streaked on MuellerHinton agar (Difco Laboratories, Inc., NJ, USA). Then, the appropriate antimicrobial-impregnated disks were placed on the surface of the agar, and the plate was incubated at $37^{\circ} \mathrm{C}$ for 18-24 hr. Thereafter, we measured the zones of inhibition and determined the antimicrobial susceptibility of the isolates using interpretative standards for Staphylococcus species. The results were reported as susceptible, intermediate, or resistant $[8,9]$.

\section{Results}

Table 1 presents the prevalence of $S$. aureus contamination in pork sampled from each fresh meat market. S. aureus was detected in $28.18 \%(31 / 110)$ of the samples from the 12 markets, including $40 \%$ ( 2 of 5 , no. 1 market), $25 \%$ (5/20, no. 2 market), $35 \%$ (7/20, no. 3 market), 50\% (5/10, no. 4 market), $0 \%$ (0/5, no. 5 market), $20 \%$ (1/5, no. 6 market), $0 \%$ (0/5, no. 7 market), $0 \%(0 / 5$, no. 8 market), $40 \%(2 / 5$, no. 9 market), $40 \%$ ( $4 / 10$, no. 10 market), $20 \%$ ( $1 / 5$, no. 11 market), and $26.67 \%$ (4/15, no. 12 Market). The antimicrobial susceptibility tests are presented in Table 2 . Of the 52 isolates tested, $92.23 \%$ (49/52) were resistant to one or more antibiotics. Resistance to tetracycline and ampicillin was detected in $80.77 \%(42 / 52)$ and $76.92 \%(40 / 52)$ of isolates, respectively. Resistance to two drugs was found in $67.3 \%$ $(35 / 52)$ of isolates. Furthermore, the $S$. aureus isolates were $100 \%(52 / 52)$ susceptible to cloxacillin, cefoxitin, gentamicin, and cephazolin. Additionally, $13.46 \%$ (7/52) of isolates were scored as intermediate to vancomycin.

\section{Discussion}

In this study, the prevalence of $S$. aureus contamination in pork from 12 fresh meat markets was $28.18 \%$. One of the possibility factors involved in the contamination is "the processing of
TABle 1: Prevalence of Staphylococcus aureus contamination in pork from each fresh meat market.

\begin{tabular}{lc}
\hline No. of markets & Percentage (no. of positive total) \\
\hline 1 & $40(2 / 5)$ \\
2 & $25(5 / 20)$ \\
3 & $35(7 / 20)$ \\
4 & $50(5 / 10)$ \\
5 & $0(0 / 5)$ \\
6 & $20(1 / 5)$ \\
7 & $0(0 / 5)$ \\
8 & $0(0 / 5)$ \\
9 & $40(2 / 5)$ \\
10 & $40(4 / 10)$ \\
11 & $20(1 / 5)$ \\
12 & $26.67(4 / 15)$ \\
Total & $\mathbf{2 8 . 1 8 ( 3 1 / 1 1 0 )}$ \\
\hline
\end{tabular}

meat in slaughterhouse" which includes many steps of risk to expose microorganisms such as washing the carcass and portioning of carcass using cutting equipment. The second possibility of contamination is "the locations of fresh meat markets" in the present study which are open area and being nearby streets, car parking, and car transportation with heavy traffic. A recent study reported the occurrence of pathogenic bacterial strains in the environment at a high probability such as $S$. aureus which can survive under unfavorable conditions on dry surfaces for a long period, even several months [10]; therefore, the contamination of equipment and hygiene (such as a knife, cutting board, apron, and hand towel) in the slaughterhouse and of fresh meat in the open air markets might occur. A previous study of bacterial contamination's prevalence in similar location to this study supports our finding. A survey reported sushi and sashimi from the flea market at Rangsit Campus, Thailand, were contaminated with S. aureus $46.88 \%$, that is, a high level of contamination [11] indicating that an open area such as fresh markets or flea markets has a chance to contaminate the pork or cooked foods. Furthermore, the consumer should clean meat before cooking and avoid eating raw meat. Another factor of bacterial contamination is the weather condition of collected sample's location. Our study investigated the pork sample in Bangkok, Thailand, which is a country of hot and humid climate, and the prevalence of $S$. aureus contamination was $28.18 \%$. In contrast, a previous study in the USA reported the percentage of pork at $43.3 \%$ which is much higher than this study. The integrated geography effects to the prevalence of $S$. aureus contamination [12].

S. aureus, a most common cause of food-borne disease, is a problem of the heath care management due to the resistance to antibiotic toward many classes of antimicrobials [13]. S. aureus is a leading cause of community-acquired and healthcare-associated bacteremia. Furthermore, S. aureus causes nosocomial infections in hospitalized patients. Tetracycline and ampicillin are effective against many Grampositive and Gram-negative bacteria and are used to treat chronic respiratory diseases $[4,14]$. However, the antimicrobial susceptibility test results of isolated $S$. aureus in our study showed a high percentage of tetracycline- and 
TABLE 2: Antibiotic susceptibility pattern of $S$. aureus isolated from pork from 12 fresh meat markets.

\begin{tabular}{lccc}
\hline Antimicrobial & $\begin{array}{c}\text { Resistant } \\
\text { (no. of positive/total isolates) }\end{array}$ & $\begin{array}{c}\text { Intermediate } \\
\text { (no. of positive/total isolates) }\end{array}$ & $\begin{array}{c}\text { Susceptible } \\
\text { (no. of positive/total isolates) }\end{array}$ \\
\hline Tetracycline & $80.77(42 / 52)$ & $1.92(1 / 52)$ & $17.31(9 / 52)$ \\
Cloxacillin & 0 & 0 & $100(52 / 52)$ \\
Cefoxitin & 0 & 0 & $100(52 / 52)$ \\
Gentamicin & 0 & 0 & $100(52 / 52)$ \\
Ampicillin & $76.92(40 / 52)$ & 0 & $23.07(12 / 52)$ \\
Cephazolin & 0 & 0 & $100(52 / 52)$ \\
Vancomycin & 0 & $13.46(7 / 52)$ & $86.53(45 / 52)$ \\
\hline
\end{tabular}

ampicillin-resistant strains similar to the previous study in pork isolates, which were highly resistant to tetracycline and ampicillin $94.8 \%$ and $100 \%$, respectively [10]. Reflecting the limited therapeutic value of these antimicrobial agents for treatment of $S$. aureus infected humans [2]. Furthermore, $13.46 \%$ of the isolates in our study were intermediate resistant to vancomycin, a drug of choice for treatment of MRSA (methicillin-resistant S. aureus) infections in hospitals [14]. This prevalence suggests that the level of resistance of $S$. aureus to vancomycin might increase in the future in Thailand, resulting in a serious global problem when treating MRSA infections in hospitals [15]. Moreover, recently in India, VRSA (vancomycin-resistant $S$. aureus) was already detected $16 \%$ from buffalo nasal and skin samples using the disk diffusion method which is a dangerous public health issue [16].

\section{Data Availability}

Previously reported (antimicrobial-resistant strain) data were used to support this study and are available at (DOI: 10.4315/0362-028X-72.3.608, DOI: $10.1111 /$ jfs.12589). These prior studies are cited at relevant places within the text as references $[2,4]$.

\section{Conflicts of Interest}

The authors declare that they have no conflicts of interest.

\section{Acknowledgments}

The authors thank the year 6 students of Faculty of Veterinary Medicine, Mahanakorn University of Technology, Thailand, for samples collection and laboratory testing. The authors also thank the Budget from Veterinarian Project for supporting this research.

\section{References}

[1] J. C. U. waezuoke and L. E. Aririatu, "A survey of antibiotic resistant Staphylococcus aureus strains from clinical sources in Owerri," Journal of Applied Sciences \& Environmental Management, vol. 8, no. 1, pp. 67-69, 2004.

[2] L. Jyhshiun, Y. Kuang-sheng, L. Hsueh-tao, and L. Jiunn-horng, "Staphylococcus aureus isolated from pork and chicken carcasses in Taiwan: prevalence and antimicrobial susceptibility," Journal of Food Protection, vol. 72, no. 3, pp. 608-611, 2015.

[3] J. S. Weese, B. P. Avery, and R. J. Reid-Smith, "Detection and quantification of methicillin-resistant Staphylococcus aureus
(MRSA) clones in retail meat products," Letters in Applied Microbiology, vol. 51, no. 3, pp. 338-342, 2010.

[4] R. S. Wilfred, B. R. Narendra, K. Porteen et al., "Prevalence, antimicrobial susceptibility and virulence genes of Staphylococcus aureus isolated from pork meat in retail outlets in India," Journal of Food Safety, vol. 39, pp. 1-8, 2018.

[5] Y. C. T. Steven, S. D. Joshua, E. Emily, L. H. Thomas, and G. F. Vance Jr., "Staphylococcus aureus infections: epidemiology, pathophysiology, clinical manifestations, and management," Clinical Microbiology Reviews, vol. 28, no. 3, pp. 603-661, 2015.

[6] Z. Feng and W. Yuyan, "Characteristic of antibiotic resistance of airborne Staphylococcus isolated from metro stations," International Journal of Environmental Research and Public Health, vol. 10, pp. 2412-2426, 2013.

[7] OIE, "Laboratory methodologies for bacterial antimicrobial susceptibility testing," OIE Terrestrial Manual, vol. 31, no. 1, pp. 1-11, 2012.

[8] A. P. Eurl, "Isolation of methicillin-resistant Staphylococcus aureus (MRSA) from food producing animals and farm environment," DTU National Food Institute, vol. 1, pp. 1-8, 2018.

[9] H. Jan, Kirby-Bauer Disk Diffusion Susceptibility Test Protocol, American Society for Microbiology, Washington, DC, USA, 2009.

[10] A. Kozajda, K. Jeżak, and A. Kapsa, "Airborne Staphylococcus aureus in different environments-a review," Environmental Science and Pollution Research, vol. 26, no. 34, pp. 34741-34753, 2019.

[11] R. Tiengtip, "Anisakis spp. parasite and Staphylococcus aureus, Bacillus cereus in sushi and sashimi from Thammasat university (Rangsit campus) area restaurants," Thammasat Medical Journal, vol. 20, no. 4, pp. 307-315, 2020.

[12] L. Abdalrahman, H. Wells, and M. Fakhr, "Staphylococcus aureus is more prevalent in retail beef livers than in pork and other beef cuts," Pathogens, vol. 4, no. 2, pp. 182-198, 2015.

[13] A. S. Hakim, M. A. Bakry, A. A. Samy et al., "Staphylococcus aureus a plublic health concern of retail pork and pork byproducts with spot on Egyptian market," Xeno Journal of Biomedical Sciences, vol. 1, no. 1, pp. 20-26, 2020.

[14] F. J. C. Millard and J. C. Batten, "Comparison of ampicillin and tetracycline in chronic bronchitis," British Medical Journal, vol. 1, no. 5331, pp. 644-646, 1963.

[15] T. Jing, H. Jiali, K. Lei, D. Zhengjun, W. Jiaofen, and P. Jiaqian, "The use of vancomycin in the treatment of adult patients with methicillin-resistant Staphylococcus aureus (MRSA) infection: a survey in a tertiary hospital in China," International Journal of Clinical and Experimental Medicine, vol. 8, no. 10, pp. 19436-19441, 2015.

[16] K. Al Amery, M. Elhariri, A. Elsayed et al., "Vancomycin-resistant Staphylococcus aureus isolated from camel meat and slaughterhouse workers in Egypt," Antimicrobial Resistance and Infection Control, vol. 8, no. 1, pp. 1-8, 2019. 